ment makes antisera and antitoxins for, inter alia, diphtheria, tetanus, gas gangrene, botulism, anthrax, and scorpion poison. In addition to studies of antitoxin production it has also a special interest in the immunopathology of trypanosomal infections and the blood-feeding habits of insect vectors of trypanosomiasis and other diseases. In the field of viral vaccines the Institute is best known for its smallpox vaccine, of which it is the largest manufacturer in the country. It has led the way in the mass production of dried heat-stable vaccine for tropical use, and recent researches give promise both of a novel smallpox vaccine inactivated photodynamically and of a rabies vaccine designed to minimize the risk of encephalitis. Also at Elstree is the large Blood Products Laboratory, established in collaboration with the Medical Research Council. There freeze-dried whole plasma, albumin, fibrinogen, thrombin, and concentrated antihaemophilic factor are prepared for use in surgery and gammaglobulins for the prevention of measles and other diseases. It is noteworthy that many of the techniques employed originated in basic research at Chelsea.

As well as these pursuits the Lister Institute has important educational functions. It is a School of London University, and the 56 graduate scientists on its staff include five professors, one reader, and seven recognized teachers. As well as accepting $\mathrm{Ph} . \mathrm{D}$. students time is devoted to training workers from overseas, particularly from developing countries, in production methods.

It is of interest that the Institute conducts all these activities without direct financial subvention from Government or university sources. For special projects it receives support from various research councils, from private donations, and from non-governmental bodies. Otherwise its finances are based on its own endowment fund and on the sale of immunological products. In this sense, then, the Lister Institute is something of an anomaly in a period when the trend toward governmental control of science is increasing. But it is clear that as well as making vaccines the Institute also makes a first-rate contribution to medical science in Great Britain. The record of its first 75 years and the continued expansion of its manifold interests give every promise of an even more productive future.

\section{Detection of Cervical Cancer}

The possibility of preventing any form of cancer deserves the urmost attention. This has become notably true of the female genital organs because methods exist for the early detection of cancer in them. But only for cancer of the uterine cervix are the methods well advanced. Cytology in the diagnosis of cancer of the corpus, though possible by examination of smears made from the posterior forniceal pool, is subject to an error of about $3 \mathrm{~J} \%$ and so is not usually deemed to be sufficiently exact for routine use. It has been suggested that cytological study of ovarian tumours is possible by aspirating material from the pouch of Douglas, but little is yet known of the value and dangers of this procedure. For practical purposes at present only cervical cytology seems to be of real value in detecting preinvasive carcinoma and lesions which have only just begun to invade the underlying stroma. Colposcopy and colpomicroscopy ${ }^{12}$ may be used as adjuncts to cytology, but since they are time-consuming and have some technical difficulties they have not gained general acceptance.
Cancer of the cervix uteri kills about 2,500 women every year in England and Wales, cancer of the corpus about 1,000, cancer of the ovary about 3,000, and cancer of the breast about 10,000, while cancer of the alimentary tract causes about 15,000 deaths of women. Though these statistics do not diminish the need for detecting cervical cancer in its early stages, they do show that other areas besides the cervix require attention. This is a point made by Professor T. N. A. Jeffcoate in his article at page 1091 of the B.M.F. this week. Palpation of the breast in a population-screening programme may be as valuable as cervical cytology in preventing the deaths of women from malignant disease. This points to one of the shortcomings of screening programmes based on the use of relatively medically unskilled helpers who have had special training simply to take cervical smears, and even more does it show how the taking of smears by patients themselves may fail to detect important lesions elsewhere. Ideally this means that in the detection of early cancer, whatever its site, doctors should do the work. Obviously this may not be feasible, and programmes have to be tailored to suit the resources available. A further point of Jeffcoate's is that florid cervical cancer may easily be missed if reliance is placed on cytology alone, and this applies to other pelvic lesions which may be diagnosed only by careful clinical examination.

The pathologist has an onerous duty in the examination of cervical smears. As in so much else, the normal and the grossly abnormal may be easily recognized. It is the doubtful smear that causes the difficulty. The pathologist who reports many smears as doubtful may cause much unnecessary distress to patients, as well as wasting valuable inpatient hospital facilities when cone biopsy is performed, as it must be in such cases. In addition he increases the work of the histological department. This again argues for the cytologist and histologist to be the same person, for only so will the cytologist keep an adequate check on himself. J. Elizabeth Macgregor and Sir Dugald Baird ${ }^{3}$ have suggested that to detect a cancer of the cervix in its early stages costs about £60. A poor pathological service would inflate this figure.

It seems well established that carcinoma of the cervix is commoner than average in women of low social class and high parity, and it is just these who are the most difficult to persuade to have routine examinations when they feel well, because of their home commitments and their failure to appreciate the importance of prevention. ${ }^{4}$ Since almost all women have come to accept antenatal care, Jeffcoate suggests that a good time for routine cytological examination of the cervix is during pregnancy. This may well be true, though the cytological changes of pregnancy increase the difficulties of diagnosis for the pathologist. But if a smear from a pregnant woman is examined, and found to be positive or doubtful, Jeffcoate's warning not to proceed immediately to cone biopsy should be especially heeded, and he counsels waiting till after the pregnancy before biopsy is done. There are other ways of getting women to come to clinics for the prevention and detection of cancer-for instance, through general practitioners or local health authorities ${ }^{5}$ - but circumstances will vary according to local drive and initiative.

Will a widespread screening programme for the detection

\footnotetext{
1 Coppleson, M., 9. Obstet. Gynaec. Brit. Emp., 1960, 67, 11. Bton, B., and Vince, S. W., F. Obstet. Gynaec. Brit. Cwlth, 1961, 68,

- Macgregor, J. E., and Baird, D., Brit. med. J., 1963, 1, 1631.

Ibid. 1965, 1, 1327.

Boyes, D. A., Fidler, H. K., and Lock, D. R., Brit. med. F., 1962,
} 
of early cervical cancer prevent deaths from this disease ? D. A. Boyes and colleagues ${ }^{6}$ reported a fall in the incidence of invasive cancer of the cervix from 28.4 to 19.7 per 100,000 in British Columbia after a large-scale screening programme, but the eradication of the disease as a cause of death remains a pipe-dream at present owing to the difficulties of applying on a mass scale a series of technical procedures that demand considerable skill. Especially to be noted too are the differences in malignancy of cervical cancers, as of all other cancers. Probably not all cervical cancers go through a detectable stage of preinvasion, some preinvasive cancers may regress, and some are incurable with present methods whatever the stage at which the cancer is detected. This is not counsel of despair nor does it lead to diagnostic and therapeutic nihilism. It is a recognition that miracles are unlikely, and Jeffcoate has done yet another service by drawing attention again to the inadequacies of a programme devoted only to cervical cytology. His article is a call to examine more of the patient than her cervix.

\section{Perseveration}

The term "perseveration" is best defined as the "continuation or recurrence of an experience or activity without the appropriate stimulus," though it has been used with other meanings. As Dr. R. S. Allison points out in his two Croonian lectures published in this and last week's issues of the B.M.F., it may be an important sign of disease in the nervous system.

The unnecessary repetition of a given movement is sometimes called motor perseveration, while inability to relax a part of the body after the performance of a movement has been called tonic perseveration. A physiological definition given by $\mathrm{H}$. H. Jasper ${ }^{1}$ is also helpful. He suggested that perseveration implied "the tendency of a set of neurones once excited to persist in the state of excitement autonomously, showing resistance to any change in this state." Clinically, perseveration implies the continuation or repetition of a purposeful response which is entirely appropriate to the first of two stimuli but is inappropriate to a second stimulus which is nevertheless essential to provoke it. As Allison points out, it is only when a second stimulus, to which a very different response would be expected, results in a recurrence of the response to the first one that we can say plainly that perseveration exists. The phenomenon is involuntary in the sense that the patient cannot check or prevent it. Clinical tests include the eyes-tongue, tongueeyes test, in which the patient is asked first to shut his eyes tightly and then to put out his tongue. Perseveration is plainly present when, after responding correctly to the first request, the patient in response to the second either closes his eyes again and fails to put out his tongue, or puts out his tongue and closes his eyes simultaneously. Tests of serial drawing of different shapes, searching for hidden objects, counting aloud, naming the days of the week, repeating the names of towns or cities, or writing to dictation are also useful. Thus, in writing, the patient may repeat a part or the whole of certain words.

Though perseveration was first defined as a symptom of diffuse or focal brain disease, it may occur naturally in healthy people. It is commonest in childhood, relatively infrequent in adult life, but again mare frequent in old age. ${ }^{2}$
Attempts to determine whether perseveration in normal people can be related to personality have been on the whole unsuccessful, though $\mathrm{H}$. J. Eysenck ${ }^{3}$ considers that there may be a relationship between some perseveratory phenomena and certain personality traits. The more complex the stimulus and its required response, the more likely is perseveration to occur, while it is also commoner when stimuli are applied at short but regular intervals. Tension, anxiety, and fatigue also increase its incidence, perhaps because the subject fails to grasp sufficient details of the first stimulus to enable him to perceive that the second one is different.

Allison records that for the past 15 years he has been searching as a routine for perseveration in a wide variety of organic brain conditions, ranging from acute and chronic diffuse afflictions to focal lesions, whether due to a tumour or to vascular disease. It is not common in neurotic patients, though other workers have found it to be a common feature of certain psychoses. Allison finds that it most often occurs in states of disturbed consciousness, and may pass unnoticed when there are other more obvious symptoms. It may complicate the phase of disorientation that follows head injury and is common during recovery from carbon-monoxide poisoning. It may also accompany early intellectual deterioration in presenile dementia and cerebral atherosclerosis, while in cases of acute or subacute vascular and neoplastic lesions in either cerebral hemisphere it usually indicates increasing cerebral oedema. Metabolic disorders, including cerebral anoxia, drug intoxication, electrolyte imbalance, hypoglycaemia, and hepatic encephalopathy may all produce it. Allison stresses that inexperienced doctors and nurses usually have no difficulty in recognizing profound clouding of consciousness but can easily be misled into mistaking the incongruous behaviour of perseveration for hysteria.

The perseveration itself can rarely, if ever, be used as a localizing sign of a focal brain lesion. Some of the peculiar disorders of movement which result from damage to the frontal lobes are allied to perseveration and may represent a motor manifestation of it. Perhaps-involuntary grasping and groping may be so regarded. However, it seems that perseveration is rarely the result of a unilateral frontal lesion and it almost always implies bilateral brain damage. By contrast, it is not uncommon in lesions of the parietal lobes. Here the patient may feel repeated contacts or sensory stimuli when there are none, while the visual after-images which are sometimes noted in patients with lesions of the parieto-occipital region may also be regarded as perseveratory phenomena. Perseveration in speech is of doubtful import. Occasionally a patient's speech is contaminated "by words which he has already used but cannot get rid of." But most writers on aphasia conclude that perseveration in speech is not a part of aphasia and is generally due to associated confusion rather than to the lesion causing the speech disorder. Nevertheless, Allison, after looking carefully for it in 24 patients with dysphasia, found that it was observed repeatedly in 16 of them, and that it might persist for weeks, months, or even for years. None of the 16 patients perseverated on each and all of the tests given, and all but five did so only in activities requiring a special use of speech and language. There was a striking relationship between perseveration and the absence of spontaneous talk in 13 our of 16 patients, and Allison concluded that there seems to be

\footnotetext{
Jasper, H. H., Ұ. soc. Psychol., 1931, $2,28$.

Cattell, R. B., f. ment. Sci., 1935, 81, is1.

Eysenck. H. J.. The Jrructure of Human Personality, 1953. London.

- Brain, W. R., Brain, 1961, 84, 145.
} 